\title{
Erratum to: Higgs boson production at hadron colliders: hard-collinear coefficients at the NNLO
}

\author{
Stefano Catani ${ }^{1, \mathrm{a}}$, Massimiliano Grazzini ${ }^{2, \mathrm{~b}, \mathrm{c}}$ \\ ${ }^{1}$ INFN, Sezione di Firenze and Dipartimento di Fisica e Astronomia, Università di Firenze, 50019 Sesto Fiorentino, Florence, Italy \\ ${ }^{2}$ Institut für Theoretische Physik, Universität Zürich, 8057 Zürich, Switzerland
}

Received: 1 August 2012 / Published online: 31 August 2012

(C) Springer-Verlag / Società Italiana di Fisica 2012

Erratum to: Eur. Phys. J. C (2012) 72:2013

DOI 10.1140/epjc/s10052-012-2013-2

The expression of $\mathcal{H}_{g g \leftarrow g q}^{H(2)}$ in Eq. (23) contains two mistyped signs. The typos are in the sixth line, where the second term,

$+\frac{1}{24 z}\left(\left(1+(1+z)^{2}\right)\left(3 \ln ^{2} z-\pi^{2}\right)-6 z^{2} \ln z\right) \ln (1+z)$,

has to be replaced by the following correct expression:

$-\frac{1}{24 z}\left(\left(1+(1+z)^{2}\right)\left(3 \ln ^{2} z+\pi^{2}\right)-6 z^{2} \ln z\right) \ln (1+z)$.

The online version of the original article can be found under doi:10.1140/epjc/s10052-012-2013-2.

a e-mail: catani@fi.infn.it

be-mail: grazzini@physik.uzh.ch

${ }^{\mathrm{c}} \mathrm{M}$. Grazzini is on leave of absence from INFN, Sezione di Firenze, Sesto Fiorentino, Florence, Italy. 